\title{
Development and Initial Characterization of a Staphylococcus Collection Obtained from Healthy Student Volunteers
}

\author{
By Jeremiah J. Davie*
}

\begin{abstract}
Here, we announce the availability of a collection of Staphylococci isolated from healthy student volunteers enrolled in Biology or Allied Health majors. Undergraduate students preparing for careers in healthcare or healthcare-associated fields frequently complete clinical rotations as part of their education while remaining members of the general college community. This positions them as possible sources of both communityacquired and healthcare-acquired MRSA. From Fall 2012 to Fall 2013, sampling and characterization of bacterial isolates from the anterior nasal nares or skin was performed for healthy volunteers and preliminary biochemical characterization was performed. 27 putative $S$. aureus (18\%) and 126 putative coagulase-negative Staphylococci (CoNS) (82\%) isolates were recovered. To provide an initial survey, 15 isolates from each group (20\% of the collection) were selected for additional characterization. Among putative $S$. aureus isolates, clinically significant resistance to ampicillin was widespread, yet resistance to other antibiotics was infrequent. Among putative CoNS isolates, clinically significant resistance to ampicillin and erythromycin was widespread; oxacillin resistance was infrequent. The relative paucity of colonization by oxacillin-resistant (MRSA) organisms suggests students are unlikely to be colonized prior to formal entry into their field. Notably, CoNS harbored by healthy individuals may serve as a reservoir of antibiotic resistance genes that could be spread to other organisms via lateral gene transfer. Further analysis of this collection of non-clinical isolates is ongoing and is intended to serve as a resource for the biomedical research community.
\end{abstract}

Keywords: Antibiotic resistance, CoNS, Sample collection, Staphyloccocus.

\section{Introduction}

The genus Staphylococcus is a highly clonal collection of $>40$ species of halotolerant, Gram-positive cocci associated with the skin and mucus membranes of humans and other mammals (Reviewed in Becker 2014). Historically, medical microbiologists and clinical personnel have divided this genus into two groups based on the ability to produce the virulence factor coagulase. With the exception of comparatively uncommon incidences of colonization by animal-associated strains, human coagulase positive Staphylococcal (CPS) isolates are limited to the $S$. aureus species group (Reviewed in Becker 2014). S. aureus is a colonist of the anterior nasal nares of $15-30 \%$ of the population, as well as a common inhabitant of the posterior pharynx, vagina, rectum, and perineum. S. aureus is

\footnotetext{
* Assistant Professor, Department of Biology and Mathematics, School of Arts, Science, and Education, D'Youville College, USA.
} 
a potent pathogen capable of causing skin and soft tissue infections, invasive diseases (e.g. osteomyletis, bacteremia, meningitis, and endocarditis), and a number of exotoxin-mediated diseases (e.g. Staphylococcal gastroenteritis, Staphylococcal Toxic Shock and Scalded Skin Syndrome) (Jorgensen et al., 2015; Podkowik et al., 2013).

In contrast to $S$. aureus, the coagulase-negative Staphylococci (CoNS) have been the subject of considerably less investigation, owing primarily to a persistent assumption that these organisms represented harmless commensals (Reviewed in Becker, 2014 and Namvar et al., 2014). However, CoNS species are capable of causing infectious keratitis, sepsis of patients in neonatal intensive care units, and are now being recognized as a cause of gastroenteritis (Reviewed in Podkowik et al. 2013; Dong and Speer, 2014). Recently, evidence has accumulated to suggest that lateral gene transfer from CoNS has resulted in antibiotic resistance among $S$. aureus isolates (Chan et al., 2011).

It is interesting to note that the vast majority of what we know about virulence and antibiotic resistance among the Staphylococci, both CPS and CoNS, is derived from clinical specimens (Reviewed in Becker et al., 2014). Despite high carriage rates of $S$. aureus among healthy individuals, including healthcare workers, and substantial evidence identifying the CoNS as both potent opportunistic pathogens and sources of transferable antibiotic resistance, very few studies and/or isolate collections have been prepared with Staphylococci isolated from healthy volunteers in non-clinical settings. Here, we describe the development and broad characteristics of a collection of 153 putative Staphylococci isolated from healthy college student volunteers. In addition, a selected subset of these isolates was subjected to an initial biochemical and molecular characterization, including antibiotic susceptibility assays and 16S rRNA gene sequencing.

\section{Materials and Methods}

\section{Specimen Isolation and Preservation}

Staphylococci were isolated by swabbing the anterior nasal nares or a skin location of the students' choice. The swabs were used to inoculate m-Staph Broth (mSB; Hardy Diagnostics, CA, USA) cultures and were incubated aerobically under static conditions for $24-48 \mathrm{hrs}$ at $37^{\circ} \mathrm{C}$ and then refrigerated. $\mathrm{mSB}$ is selective for Staphylococci and inhibits growth of other normal flora found in the nares (Jorgensen et al., 2015). Refrigerated cultures were then used to inoculate mannitol salt agar (MSA; Becton Dickinson, NJ, USA) plates which were then incubated aerobically for $24-48 \mathrm{hrs}$ at $37^{\circ} \mathrm{C}$ and then refrigerated to identify strains capable of mannitol fermentation. MSA plate cultures were used to inoculate blood agar (BA; Hardy Diagnostics) plates which were incubated for overnight aerobically at $37^{\circ} \mathrm{C}$ and then refrigerated until observed. Specimens for which permission was granted to keep and record data from were then inoculated to $\mathrm{mSB}$ and grown as described to 
inhibit contaminates prior to cryopreservation in Trypticase Soy Broth (TSB; Hardy Diagnostics) supplemented to a final concentration of $15 \%$ glycerol. Cryopreserved specimens were held at $-80^{\circ} \mathrm{C}$ indefinitely.

\section{Specimen Preservation Criteria}

Students enrolled in the Microbiology laboratory from Fall 2012 to Fall 2013 were given the option of participating in a research study by donating bacterial specimens isolated from their person in addition to a small quantity of associated biographical and biological information. Participation in sample collection was strictly voluntary, and all data were handled confidentially and anonymously. All participants signed an informed consent form in addition to providing the following biographical data: age, sex, major, ethnicity, and site of specimen isolation. Furthermore, biological data were supplied by the student for an isolated specimen with respect to: Gram reaction, mannitol fermentation, growth in $\mathrm{mSB}$ and on MSA plates, and hemolysis activity on BA plates. These data were used by the author to make presumptive species identifications for each isolate. This study was conducted with the approval of the D'Youville College Institutional Review Board.

\section{Presumptive Species Identifications}

Putative species identifications were made solely on the basis of student supplied biochemical data obtained for their donated bacterial isolate during laboratory exercises. An identification of $S$. aureus was made for any strain identified as being Gram-positive cocci in clusters, mannitol fermentation positive, and $\beta$-hemolytic on blood agar (Hardy Diagnostics). Gram-positive cocci in clusters with any other combination of mannitol fermentation and hemolysis activity observation (excluding $\alpha$-hemolysis) were identified as CoNS (Jorgensen et al., 2015).

\section{Antibiotic Sensitivity Profiling}

Kirby-Bauer radial diffusion assays were performed in accordance with Clinical Laboratory Standards Institute (CLSI) guidelines for a selected group of isolates (Cockerill and Clinical and Laboratory Standards Institute, 2011). Briefly, overnight cultures of a selected group of putative Staphylococcus isolates were grown aerobically at $37^{\circ} \mathrm{C}, 225 \mathrm{rpm}$, in Mueller-Hinton II (MHII; Hardy Diagnostics) broth and used to inoculate day cultures by diluting the overnight cultures $1 / 20$ in fresh media. Day cultures were grown at $37^{\circ} \mathrm{C}, 225 \mathrm{rpm}$, until they reached a turbidity equivalent to a $0.5 \mathrm{MacFarland}$ standard. Day cultures were then used to prepare confluent lawns of each specimen on MHII agar. After allowing 20 minutes for excess media to be absorbed, commercially prepared antibiotic disks (Becton-Dickinson) were placed on the surface of the agar plate. All plates were incubated aerobically for $18 \mathrm{hrs}$ at $37^{\circ} \mathrm{C}$ and zones of inhibition were measured immediately upon removal from the incubator. 
Antibiotics tested: Ampicillin $(10 \mu \mathrm{g})$, Ciprofloxacin $(5 \mu \mathrm{g})$, Erythromycin $(15 \mu \mathrm{g})$, and Oxacillin $(1 \mu \mathrm{g})$.

\section{Coagulase Production Assay}

Strains were assayed for the production of coagulase enzyme using the method described in (Finegold et al., 1978). Briefly, $100 \mu 1$ of overnight culture was used to inoculate $500 \mu \mathrm{l}$ of rabbit plasma-EDTA (Becton-Dickinson) and incubated without aeration for 4 hours at $37^{\circ} \mathrm{C}$. After 4 hours, each tube was assessed for evidence of plasma coagulation.

\section{$16 S$ rRNA Gene Sequencing}

Genomic DNA was isolated from each strain using the GeneJet Genomic DNA (gDNA) purification kit (ThermoFisher Scientific, USA) as per manufacturer's instructions. The 16S rRNA gene was amplified by PCR using the Phusion Green high-fidelity polymerase (ThermoFisher Scientific, USA) using the forward primer S-D-Bact-0008-a-S-16 (5'- AGA GTT TGA TCM TGG C -3') and reverse primer S-D-Bact-1492-a-A-16 (5'- TAC CTT GTT ACG ACT T -3') synthesized by IDT DNA technologies (IA, USA). These primers were selected from the ProbeBase primer database (http://probebase. csb.univie.ac.at/) as universal primers for the amplification of the 16S rRNA gene of $77.1 \%$ of all bacterial phyla, including the Staphylococci (Muyzer G., et al., 1995; Loy et al., 2007; Klindworth et al., 2013). Among isolates tested in this study, this primer pair yielded a PCR amplicon of $\sim 1500 \mathrm{bp}$. Amplicons were then purified using the GeneJet PCR purification kit (ThermoFisher Scientific, USA) prior to quantification via UV-Vis spectroscopy using an Biophotometer D30 (Eppendorf, NY, USA) and then sent to Eurofins Genomics (Eurofins MWG Operon LLC, KY, USA) for traditional Sanger sequencing.

\section{Bioinformatics}

Sequence data returned from Eurofins Genomics was analyzed using the following programs as implemented in the MacVector bioinformatics suite, version 14.5.3 (MacVector Inc, NC, USA). All sequences were analyzed by the Phred package to determine base-call quality values that were used by the Phrap package to inform the assembly of multiple sequence reads and were exported as nucleic acid FASTA files. The sequences in these files were then compared against the EzTaxon database using the EzTaxon-e program as implemented at: http://www.ezbiocloud. net/eztaxon/database (Kim et al., 2012). Species identification was assigned for the best-hit result from this database. In all cases, the submitted sequence (query) coverage exceeded $96 \%$ of the subject sequence record and $>99 \%$ pairwise-similarity existed between query and subject records. 


\section{Results}

\section{Description of Bacterial Specimen Donors}

Between the Fall of 2012 and the Fall of 2013, students enrolled in a microbiology laboratory course at a small college in Western New York State, USA, performed a routine laboratory exercise intended to highlight the differences between the genera Staphylococcus and Streptococcus. As part of the exercise, the students would perform Gram stain, mannitol fermentation, and hemolysis assays in order to putatively identify their isolate as Staphylococcus aureus or as a member of the coagulase-negative Staphylococci (CoNS). Following the completion of this exercise, the students were provided with informed consent regarding a request for permission by the author to preserve the bacterial specimens they had isolated from their person or belongings and subsequently analyzed as part of their laboratory exercise. Of these, 209 healthy student volunteers donated a bacterial isolate, phenotypic data pertaining to the isolate, and personal biographical data as described in the Materials and Methods section.

Among the 209 isolates donated, 153 had been directly isolated from the students nasal passages or skin; these isolates and data sets were selected for further analysis. Analysis of the age and sex of the specimen donors (Table 1) identified a distinct bias towards female donors, whose samples represent $69 \%$ of the collection. Additional analysis of the study population identifies a similarly uneven distribution when the ethnic background (Table 2) or academic major (Table 3) of the donor was taken into account. Students that self-identified as "White" or "Caucasian" represent the vast majority of individuals that consented to sample preservation and data collection. Microbiology is a required course for students enrolled in academic majors associated with the Allied Health fields and serves as a pre-requisite course for entry into several graduate or professional schools in the life sciences. Accordingly, data sets collected from Nursing $(N=70)$ and Biology $(N=45)$ majors greatly outnumbered those obtained from students enrolled in other academic majors (Table 3 ).

Table 1. Age and Sex Distribution of the Specimen Donors

\begin{tabular}{cccc}
\hline & $N$ & Median Age (years) & Age Range (years) \\
\hline \hline Males & 45 & 22 & $19-40$ \\
Females & 108 & 20 & $18-36$ \\
Total & 153 & 20 & $18-40$ \\
\hline \hline
\end{tabular}

Table 2. Self-Identified Ethnic Background of Specimen Donors

\begin{tabular}{lccc}
\hline Ethnicity & $N$ & Males & Females \\
\hline \hline White or Caucasian & 127 & 35 & 92 \\
Asian & 7 & 5 & 2 \\
African-American or African & 6 & 2 & 4 \\
Hispanic or Latino & 9 & 2 & 7 \\
Not Disclosed or Other & 4 & 1 & 3 \\
\hline \hline
\end{tabular}


Vol. 4, No. $4 \quad$ Davie: Development and Initial Characterization of a Staphylococcus...

Table 3. Academic Major Distribution of Specimen Donors

\begin{tabular}{lccc}
\hline Academic Major & $N$ & Males & Females \\
\hline \hline Biology & 45 & 15 & 30 \\
Nursing & 70 & 14 & 56 \\
Physician Assistant & 19 & 7 & 12 \\
Occupational Therapy & 1 & 0 & 1 \\
Chiropractic & 10 & 7 & 3 \\
Dietetics & 7 & 2 & 5 \\
Not Disclosed & 1 & 0 & 1 \\
\hline \hline
\end{tabular}

Description of Bacterial Isolates

Analysis of the student-supplied strain data revealed that the majority of specimens obtained directly from human tissue were nasal isolates. This trend persisted across all academic majors (Figure 1) and was common to donors of both genders (Figure 2). These results demonstrate that nasal specimens recovered from female nursing majors represent the greatest single component of the collection.

Figure 1. Distribution of Specimen Source by Body Location and Academic Major

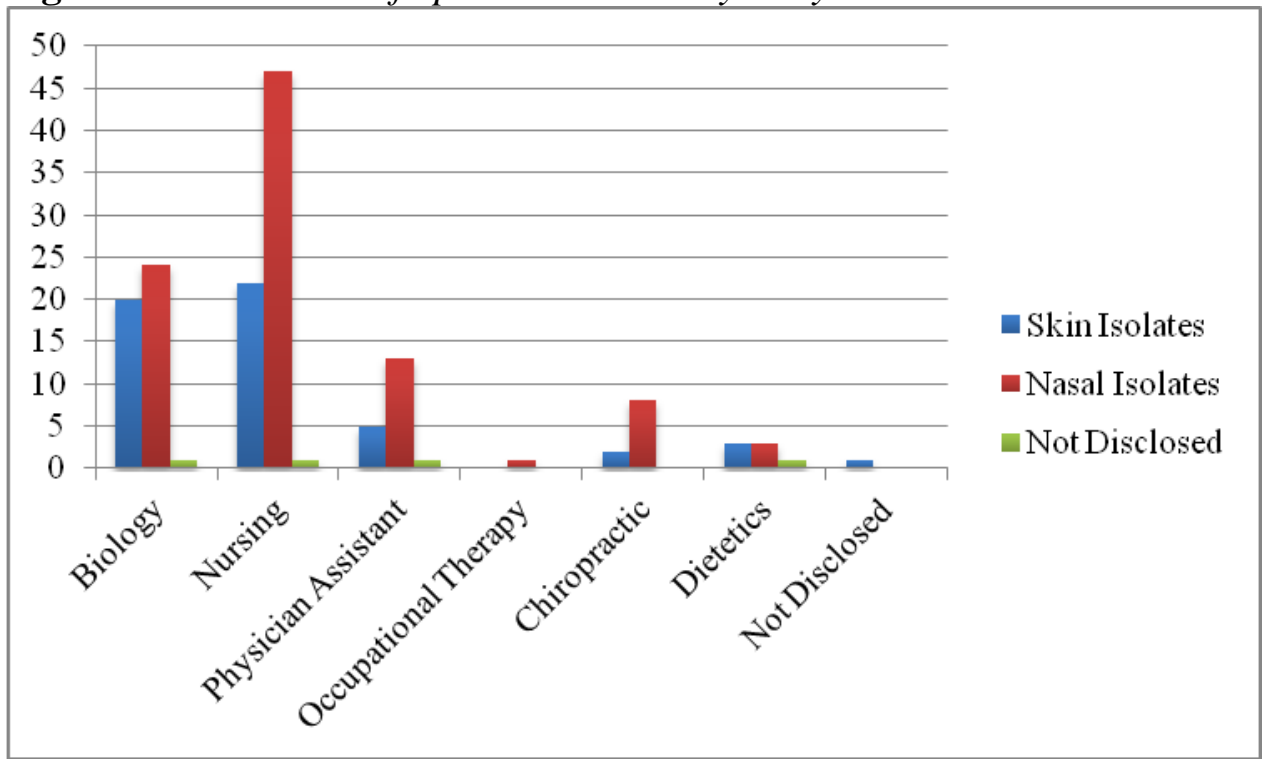


Figure 2. Distribution of Specimen Source by Donor Body Location and Sex

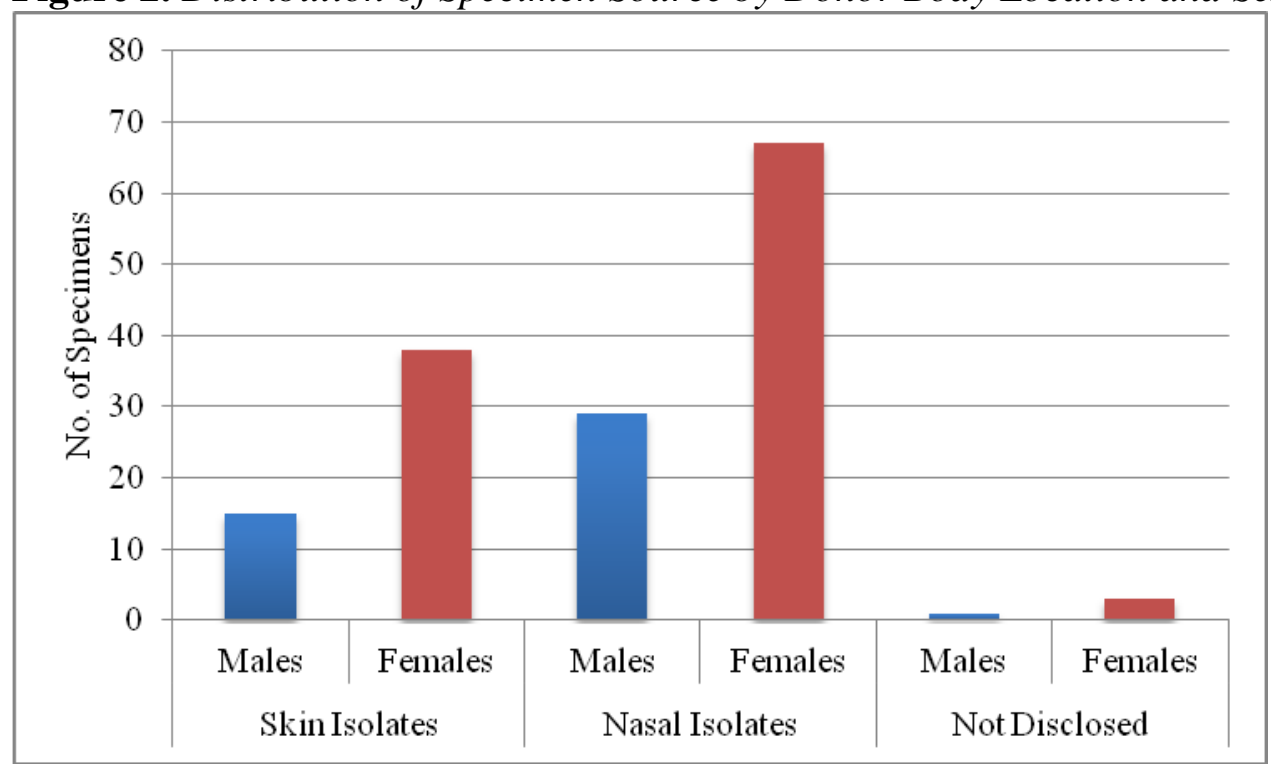

Review of the associated, student-supplied biochemical data suggested that 27 of the $153(18 \%)$ recovered isolates should be putatively identified as $S$. aureus. The remaining 126 were reported to exhibit characteristics consistent with species of the coagulase-negative Staphylococci (CoNS; 82\%) (Reviewed in Jorgensen et al., 2015; Becker et al., 2014). The distribution of these isolates by body location of isolate recovery and by academic major of the donor are found in Figures 3 and 4, respectively. Colonization by putative CoNS was observed more frequently than by putative $S$. aureus and is independent of both the location of isolation and the donors major of study. The putative CoNS represented $82.3 \%$ of all nasal isolates and $83.0 \%$ of all skin isolates recovered. Nasal carriage of putative $S$. aureus isolates was observed more frequently (17/27: 63.0\%) than skin carriage (9/27; 33.3\%). Among the two major isolate groups, e.g. specimens donated by Nursing or Biology majors, putative $S$. aureus isolates represented $24.4 \%$ and $17.7 \%$ of the donated isolates, respectively. These data are consistent with prior observations (Reviewed in Jorgensen et al., 2015; Becker et al., 2014; Nyasulu et al., 2016) that $S$. aureus colonizes the nasal passages of $15-30 \%$ of adults. 
Figure 3. Distribution of Putative Staphylococci by Location

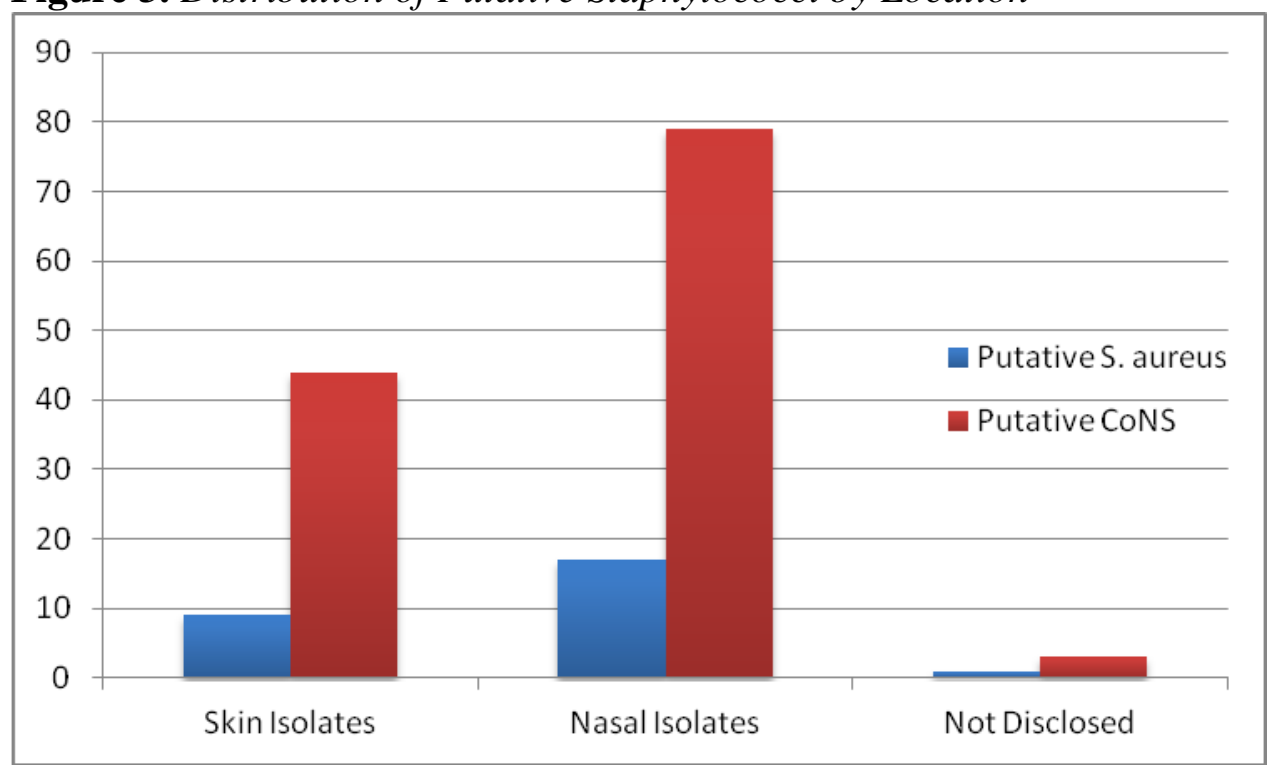

Figure 4. Distribution of Putative Staphylococci by Academic Major



\section{Biochemical and Molecular Characterization of a Subset of Isolates}

A subset containing 15 presumptive $S$. aureus and 15 presumptive CoNS isolates, representing roughly $\sim 20 \%$ of the collection, was selected for initial biochemical and molecular characterization (Table 4). The results of the authors' biochemical testing largely agreed with the student-supplied data, as only 7 of 60 observations $(11.6 \%)$ of hemolysis or coagulase activity were not in agreement with classical depictions of $S$. aureus or the CoNS. However, the high degree of genomic plasticity amongst some members of the genus rendered the use of these simple biochemical tests insufficient for distinguishing among many of the recognized species of Staphylococcus (Reviewed in Jorgensen et 
al., 2015; Becker et al., 2014). To address this, molecular analysis methods were employed to perform species identification.

16S rRNA gene sequencing has been employed previously to determine identity of Staphylococcal species (Takahashi et al., 1999; Petti et al., 2008). For each of the 30 previously selected bacterial isolates, 16S rRNA gene amplicons were purified, sequenced, and compared against the collection of $>64,000$ curated 16S rRNA gene sequences found in the EZ-Taxon 16S rRNA gene database (Kim et al., 2012). Among the selected isolates, 28 of 30 isolates were identified as members of the Staphylococci; the 16S rRNA gene sequences of two putative CoNS isolates belonged to the genus Bacillus and were excluded from further analysis (Table 4). The distribution of species identifications for the 28 sequence confirmed Staphylococci is illustrated in Figure 5. Among these isolates, three putative $S$. aureus isolates were identified as CoNS species, reducing the pool of sequence identified $S$. aureus group strains to 12 isolates. The remaining 16 isolates, including the newly reassigned strains, were distributed unevenly among the species of the CoNS. Consistent with prior clinical studies, the most commonly recovered CoNS isolates were $S$. epidermidis group members (Reviewed in Becker et al., 2014). Gene sequence data for the 28 confirmed Staphylococcal can be found in Figure 6.

Figure 5. Distribution of Selected Isolates from the Collection by $16 S$ rRNA Gene Sequencing

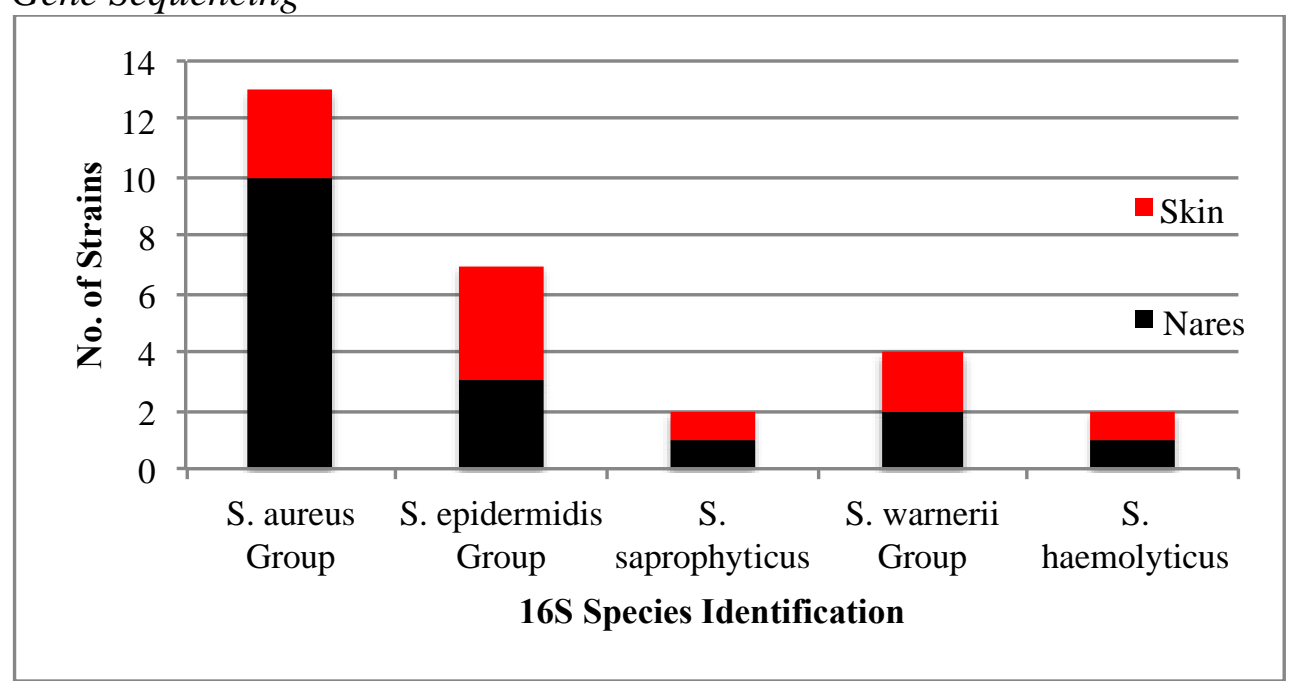


Figure 6. PCR Amplicon Sequence Data used for 16S rRNA Gene-based Species Identification for the 28 Staphylococci in this Study

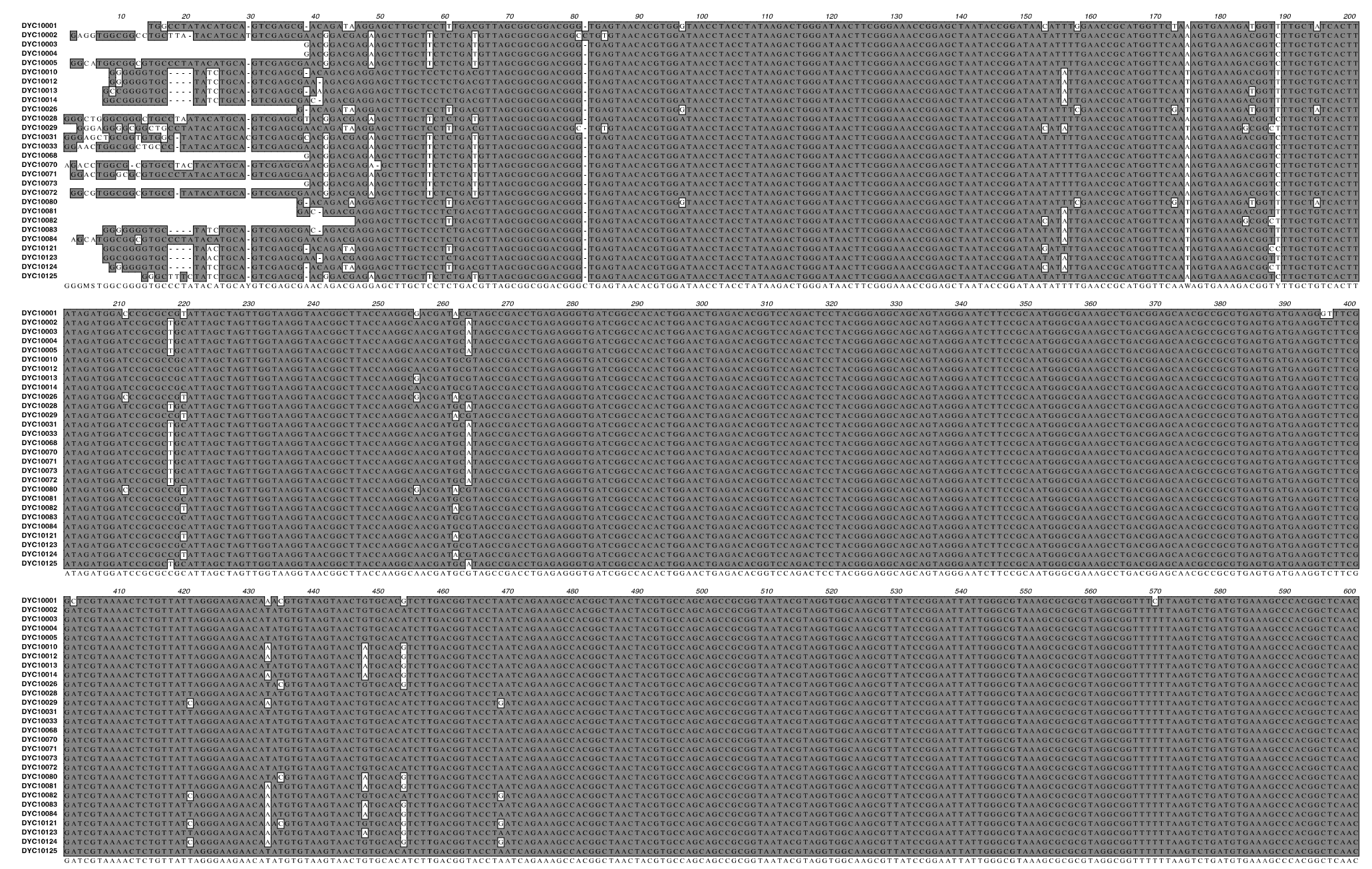




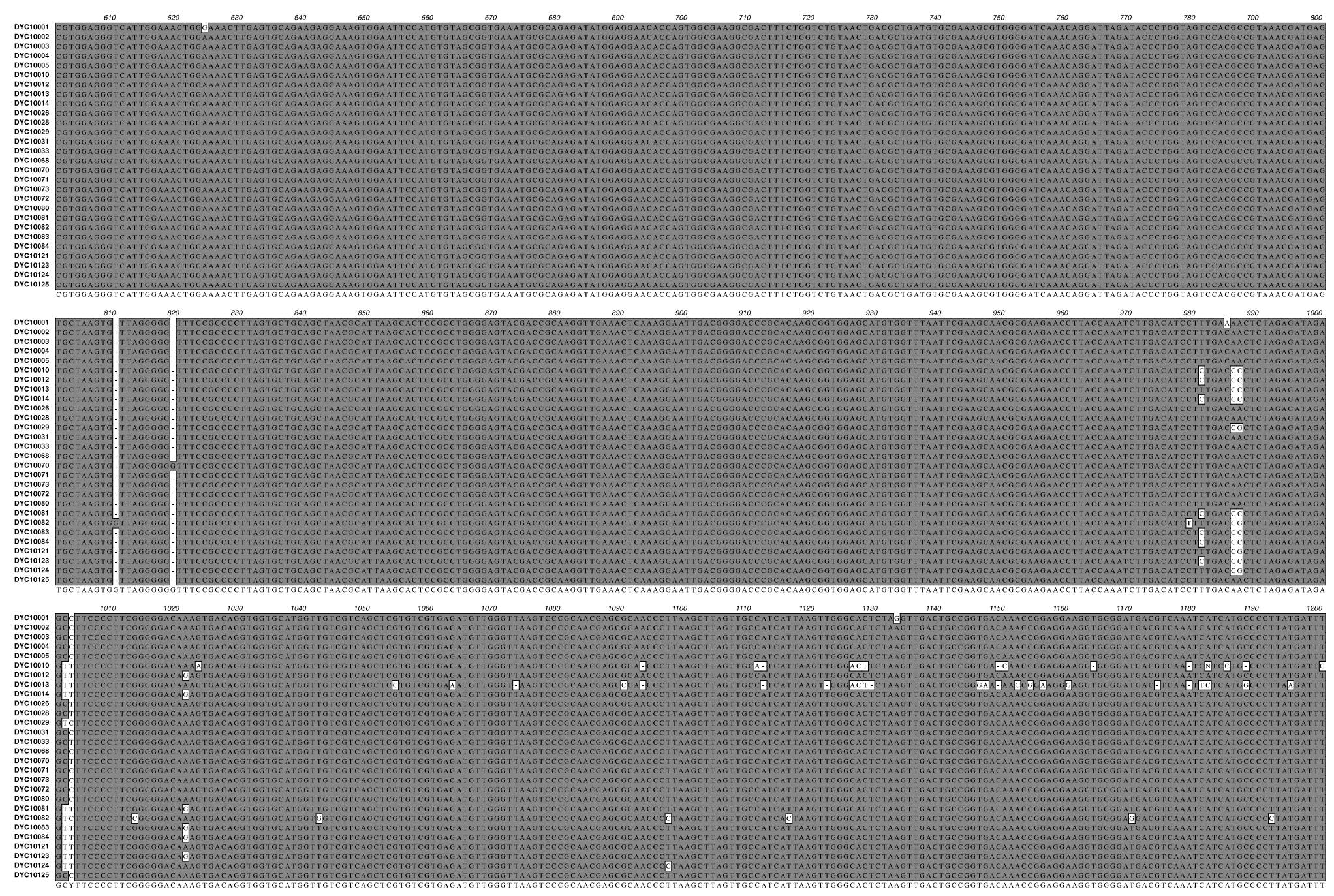



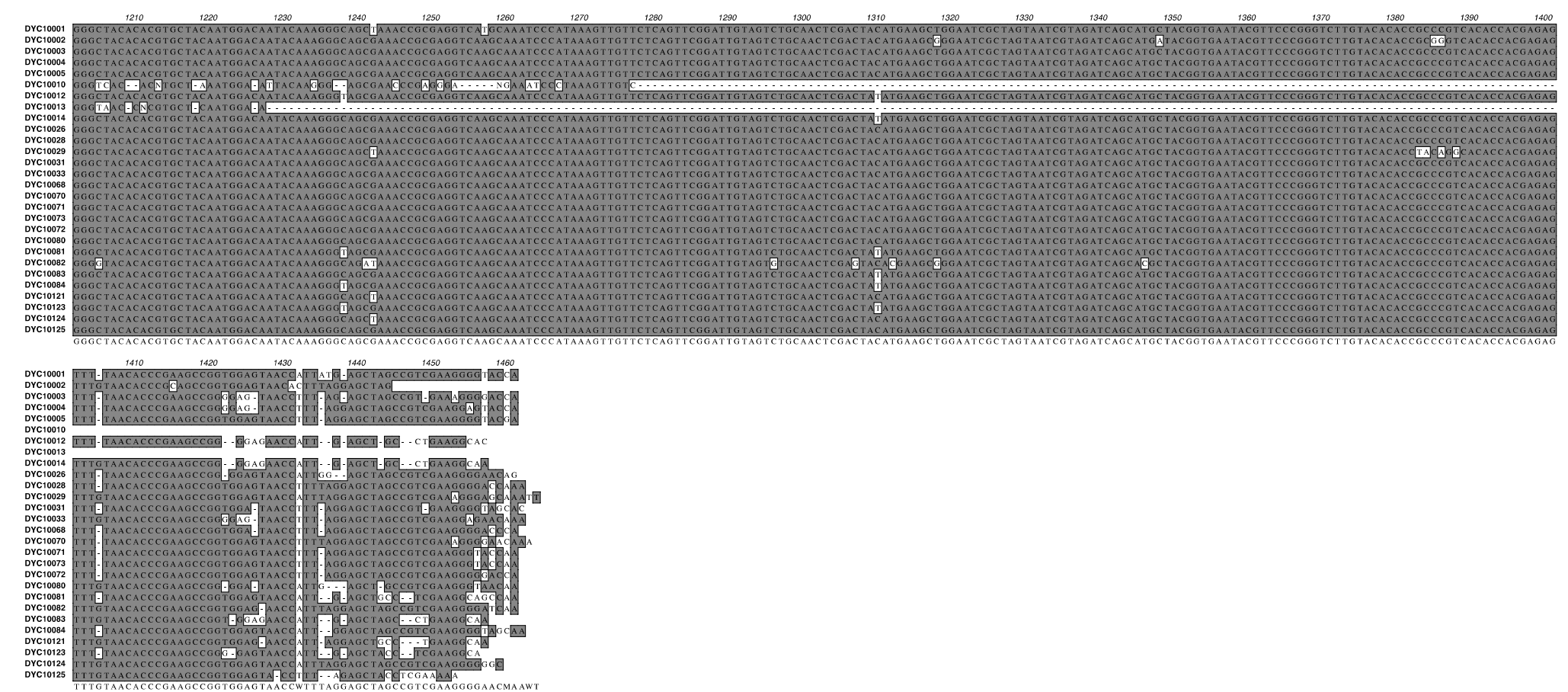
Table 4. Classification of Selected Bacterial Isolates via Biochemical and Molecular Assays

\begin{tabular}{|c|c|c|c|c|}
\hline Strain & $\begin{array}{c}\text { Location of } \\
\text { Isolation } \\
\end{array}$ & $\begin{array}{c}\text { Hemolysis } \\
\text { Activity }\end{array}$ & $\begin{array}{l}\text { Coagulase } \\
\text { Production } \\
\end{array}$ & Species Identification (16S rRNA) \\
\hline$\overline{\overline{\text { DYC10001 }}}$ & Skin & Gamma $^{\alpha}$ & Negative $^{\alpha}$ & S. saprophyticus subsp. bovis \\
\hline DYC10002 & Nares & Beta & Positive & S. argenteus ${ }^{\gamma}$ \\
\hline DYC10003 & Nares & Beta & Positive & S. aureus subsp. anaerobius \\
\hline DYC10004 & Nares & Beta & Positive & S. aureus subsp. anaerobius \\
\hline DYC10005 & Nares & Beta & Positive & S. aureus subsp. anaerobius \\
\hline DYC10010 & Skin & Gamma & Negative & S. epidermidis \\
\hline DYC10011 & Nares & Gamma & Negative & Bacillus species ${ }^{\beta}$ \\
\hline DYC10012 & Nares & Gamma & Negative & S. epidermidis \\
\hline DYC10013 & Nares & Beta & Negative $^{\alpha}$ & S. epidermidis \\
\hline DYC10014 & Nares & Beta & Negative $^{\alpha}$ & S. epidermidis \\
\hline DYC10026 & Skin & Beta & Negative $^{\alpha}$ & S. haemolyticus \\
\hline DYC10028 & Skin & Beta & Positive & S. aureus subsp. anaerobius \\
\hline DYC10029 & Skin & Gamma $^{\alpha}$ & Negative $^{\alpha}$ & S. warneri \\
\hline DYC10031 & Nares & Beta & Positive & S. aureus subsp. anaerobius \\
\hline DYC10033 & Skin & Beta & Positive & S. aureus subsp. anaerobius \\
\hline DYC10068 & Skin & Beta & Positive & S. aureus subsp. anaerobius \\
\hline DYC10070 & Nares & Beta & Positive & S. aureus subsp. anaerobius \\
\hline DYC10071 & Nares & Beta & Positive & S. aureus subsp. anaerobius \\
\hline DYC10072 & Nares & Beta & Positive & S. aureus subsp. anaerobius \\
\hline DYC10073 & Nares & Beta & Positive & S. aureus subsp. anaerobius \\
\hline DYC10080 & Nares & Beta & Negative & S. haemolyticus \\
\hline DYC10081 & Skin & Gamma & Negative & S. epidermidis \\
\hline DYC10082 & Skin & Gamma & Negative & S. warneri \\
\hline DYC10083 & Skin & Gamma & Negative & S. epidermidis \\
\hline DYC10084 & Skin & Gamma & Negative & S. epidermidis \\
\hline DYC10121 & Nares & Gamma & Negative & S. pasteuri ${ }^{\delta}$ \\
\hline DYC10122 & Nares & Gamma & Negative & Bacillus species ${ }^{\beta}$ \\
\hline DYC10123 & Nares & Gamma & Negative & S. epidermidis \\
\hline DYC10124 & Nares & Gamma & Negative & S. pasteuri ${ }^{\delta}$ \\
\hline DYC10125 & Nares & Gamma & Negative & S. argenteus ${ }^{\gamma}$ \\
\hline
\end{tabular}

$\overline{\bar{a} \text { Denotes observations that are inconsistent with the putative strain identification made with }}$ student volunteer-supplied data.

${ }^{\beta}$ Bacteria of the genus Bacillus are flagged by Ez-Taxon as difficult to distinguish by $16 \mathrm{~S}$ rRNA sequencing; these isolates are only reported at the genus level to account for that uncertainty.

${ }^{\gamma}$ Members of $S$. aureus group.

${ }^{\delta}$ Members of $S$. warnerii group.

\section{Antibiotic Resistance among Selected Bacterial Isolates}

Following CLSI guidelines, the selected bacterial isolates described above were assayed for their resistance to a modest panel of antibiotics using the Kirby-Bauer disk diffusion assay (Table 5). 11 of the 13 (85\%) of the $S$. aureus 
isolates demonstrated resistance to the beta-lactam antibiotic ampicillin. Resistance among $S$. aureus isolates to the flouroquinolone antibiotic ciprofloxacin and the macrolide antibiotic erythromycin was comparatively rare, with only $0 / 13$ and 2/13 (15\%) isolates demonstrating resistance, respectively. Resistance to oxacillin, a stand-in for the front-line beta-lactam antibiotic methicillin, was very rare and occurred in only 1 isolate.

In contrast, antibiotic resistance among the CoNS isolates tested revealed a nearly universal resistance to ampicillin $(8 / 8 ; 100 \%)$ and erythromycin $(7 / 8 ; 88 \%)$ among $S$. epidermidis group isolates. Resistance to oxacillin was non-existent among the $S$. epidermidis group isolates. Among the non-epidermidis group CoNS isolates, clinically significant resistance to oxacillin was seen in 2 of 16 (13\%) isolates, both of which were isolates of the $S$. warnerii group. Resistance to ampicillin and erythromycin among non-epidermidis group CoNS isolates was commonplace, with 4/7 (57\%) and 6/7 (86\%) of isolates resistant, respectively. No resistance to ciprofloxacin was seen among any CoNS isolate.

Table 5. Antibiotic Resistance among Selected Staphylococcal Isolates

\begin{tabular}{|c|c|c|c|c|c|c|}
\hline \multirow[b]{2}{*}{$\begin{array}{l}\text { Antibiotic } \\
\text { Disk }\end{array}$} & \multirow[b]{2}{*}{$\begin{array}{c}\text { Zone } \\
\text { of } \\
\text { Inhibition }\end{array}$} & \multicolumn{5}{|c|}{$\begin{array}{l}\text { No. of isolates identified by 16s rRNA gene sequence as } \\
\text { Staphylococcus: }\end{array}$} \\
\hline & & $\begin{array}{c}\text { aureus } \\
\text { group } \\
(N=13)\end{array}$ & $\begin{array}{c}\text { epidermidis } \\
\quad(N=8)\end{array}$ & $\begin{array}{l}\text { haemolyticus } \\
\quad(N=2)\end{array}$ & $\begin{array}{c}\text { warnerii } \\
\text { group } \\
(N=4)\end{array}$ & $\begin{array}{l}\text { saprophyticus } \\
\quad(N=1)\end{array}$ \\
\hline \multirow{2}{*}{$\begin{array}{l}\text { Ampicillin } \\
(10 \mu \mathrm{g})\end{array}$} & $\leq 28 \mathrm{~mm}$ & 11 & 8 & 1 & 3 & 0 \\
\hline & $\geq 29 \mathrm{~mm}$ & 2 & 0 & 1 & 1 & 1 \\
\hline \multirow{2}{*}{$\begin{array}{c}\text { Ciprofloxacin }(5 \\
\mu \mathrm{g})\end{array}$} & $\leq 15 \mathrm{~mm}$ & 0 & 0 & 0 & 0 & 0 \\
\hline & $\geq 16 \mathrm{~mm}$ & 13 & 8 & 2 & 4 & 1 \\
\hline \multirow{2}{*}{$\begin{array}{l}\text { Erythromycin } \\
\quad(15 \mu \mathrm{g})\end{array}$} & $\leq 13 \mathrm{~mm}$ & 2 & 7 & 1 & 4 & 1 \\
\hline & $\geq 14 \mathrm{~mm}$ & 11 & 1 & 1 & 0 & 0 \\
\hline \multirow{2}{*}{$\begin{array}{l}\text { Oxacillin } \\
(1 \mu \mathrm{g})\end{array}$} & $\leq 10 \mathrm{~mm}$ & 1 & 0 & 0 & 2 & 0 \\
\hline & $\geq 11 \mathrm{~mm}$ & 12 & 8 & 2 & 2 & 1 \\
\hline
\end{tabular}

$\overline{\text { Bolded values represent Zone of Inhibition diameters that indicate clinically significant levels }}$ of antibiotic resistance according to CLSI guidelines (Cockerill et al., 2011).

\section{Discussion}

Collections of non-clinical Staphylococcal isolates are rare. Accordingly, much of what is known regarding the pathogenic $S$. aureus group and the opportunistically pathogenic CoNS is derived from studies of clinical isolates (Reviewed in Becker et al., 2014). While epidemiological, clinical, and antibiotic resistance data for clinical specimens is important for the treatment and prevention of infection in susceptible individuals, the high degree of resistance to certain antibiotics and the potential for the movement of these resistance genes via 
lateral gene transfer within and between species of Staphylococci warrants Staphylococcal surveillance among healthy persons, especially with respect to methicillin resistance. Resistance to methicillin and related antibiotics is most commonly conferred by mecA, a gene transferred by one of 11 recognized SCCmec elements (Reviewed in Jorgensen et al., 2015). Recent studies suggest that these gene cassettes appear to have originated in animal-associated CoNS strains and are exchanged between humans and livestock in both CPS and CoNS isolates (Reviewed in Kadlec et al., 2012; Shore and Coleman, 2013). Furthermore, molecular studies of the 11 SCCmec elements suggest that the acquisition of methicillin resistance has occurred repeatedly (Reviewed in Witte et al., 2008). Much of the movement of antibiotic resistance genes between species appears to be the work of mobilizeable plasmids and transposons; Staphylococcal bacteriophage exhibit strict host limitations and have not been demonstrated to move antibiotic resistance genes between the CPS and CoNS (Reviewed in Deghorain and Van Melderen, 2012; Chan et al., 2011).

Students enrolled in healthcare or healthcare-associated majors are required to take a course in microbiology and its associated laboratory as part of their education and, thus, represent a unique population of individuals that are qualified to isolate characterize and donate non-clinical isolates of Staphylococcus. In addition to providing a limited panel of biochemical data on their bacterial specimen, they also provided biographical data regarding the specimens donor. Collection of biographical data was done as prior studies indicated that among immunologically normal patients, certain ethnic groups, most notably AfricanAmericans and persons of Aboriginal descent, exhibit a statistically higher likelihood of $S$. aureus infection (Reviewed in Messina et al., 2016; Ruimy et al., 2010). Unfortunately, this collection is anticipated to provide little insight to the carriage of Staphylococci among non-white/non-Caucasian individuals as $83 \%$ of specimen donors identified their ethnic identity as "white" or "Caucasian."

The isolation frequencies and locations for Staphylococcal species recovered in this study support the usefulness and utility of this collection. For example, prior studies have revealed that $S$. epidermis represents the dominate member of the human skin microbiome, serving to protect their host from infection with $S$. aureus by colonizing the surface skin of newborns within the first month of life and stimulating the innate immune response (Dong and Speer, 2014). Among skin isolates whose identity was confirmed by $16 \mathrm{~S}$ rRNA gene sequencing, $S$. epidermidis was the most frequently isolated species. Likewise, both putative and sequence-confirmed members of the $S$. aureus group member isolates colonized the nares more frequently than the skin (Figures 3 and 5, respectively), which is consistent with prior depictions of the literature (Reviewed in Becker et al., 2014).

Antibiotic resistance among the CoNS varies on a species-to-species basis (Reviewed in Dong and Speer, 2014). Szymanska et al. found that clinical isolates of $S$. hominis in their collection to be exhibit antibiotic resistance at lower rates than that of S. epidermidis or S. cohnii (Reviewed in Szymanska et al., 2011). The most common pathogenic clone of $S$. epidermidis, ST2, is commonly resistant to methicillin (Reviewed in Dong and Speer, 2014). The relative paucity of methicillin-resistant isolates (11\%) described in the subset of characterized isolates 
in this study suggests that possession of SCCmec elements may be less common among Staphylococci isolated from healthy individuals. Such results are consistent with another study of the non-clinical CoNS samples, which revealed less than $15 \%$ of isolates were methicillin-resistant (Widerstrom et al., 2011). However, antibiotic resistance profiling of all isolates in the collection will be required before this assertion.

The current study provides a modest insight into the patterns of Staphylococcal colonization of healthy persons yet much work remains to be done. Expansion of the antibiotic resistance testing and biofilm formation assays would be useful in assessing the virulence potential of theses isolates. While the role of $S$. aureus biofilm formation in virulence is well known, the ability of $S$. epidermidis to form a biofilm also enhances its ability to cause infection and makes it recalcitrant to antibiotic chemotherapy (Reviewed in Becker et al., 2014; David and Daum, 2010; Oliveira and Cerca, 2013). Furthermore, molecular characterization of the basis of the antibiotic resistance observed in this study will be of value in determining patterns of antibiotic resistance among Staphylococci colonizing healthy persons. Such efforts are planned or are in progress.

\section{Conclusions}

A new collection of $>150$ isolates of Staphylococci isolated from healthy student volunteers is now available to the scientific community. The majority of these isolates were acquired from Caucasian female students. The biochemical methods used for characterizing the collection were validated for a subset of isolates by using $16 \mathrm{~S}$ rRNA gene sequencing. Among that validated subset, clinically significant resistance was frequently observed among commonly prescribed antibiotics. However, methicillin resistance was rare. This collection is expected to be useful for comparisons against clinical isolate collections of Staphylococcus.

\section{References}

Becker, Karsten, Christine Heilmann, and Georg Peters. 2014. "Coagulase-Negative Staphylococci." Clinical Microbiology Reviews 27 (4): 870-926. doi:10.1128/ CMR.00109-13.

Chan, C. X., R. G. Beiko, and M. A. Ragan. 2011. "Lateral Transfer of Genes and Gene Fragments in Staphylococcus Extends beyond Mobile Elements." Journal of Bacteriology 193 (15): 3964-77. doi:10.1128/JB.01524-10.

Cockerill, F, and Clinical and Laboratory Standards Institute. 2011. Performance Standards for Antimicrobial Susceptibility Testing: Twenty-First Informational Supplement. Wayne, PA: Clinical and Laboratory Standards Institute.

David, M. Z., and R. S. Daum. 2010. "Community-Associated Methicillin Resistant Staphylococcus aureus: Epidemiology and Clinical Consequences of an Emerging Epidemic." Clinical Microbiology Reviews 23:616-687.

Deghorain, Marie, and Laurence Van Melderen. 2012. "The Staphylococci Phages Family: An Overview." Viruses 4 (12): 3316-35. doi:10.3390/v4123316. 
Dong, Ying, and Christian P. Speer. 2014. "The Role of Staphylococcus Epidermidis in Neonatal Sepsis: Guarding Angel or Pathogenic Devil?" International Journal of Medical Microbiology 304 (5-6): 513-20. doi:10.1016/j.ijmm.2014.04.013.

Finegold, Sydney M., William J. Martin, and Elvyn G. Scott. 1978. Bailey and Scott's Diagnostic Microbiology, Fifth Edition. Chapter 16, pgs.123-129. The C.V. Mosby Company.

Jorgensen, James H., Michael A. Pfaller, Karen C. Carroll, Guido Funke, Marie Louise Landry, Sandra S. Richter, and David W. Warnock. 2015. Manual of Clinical Microbiology, Eleventh Edition. Chapter 21, pgs. 354-382. American Society of Microbiology.

Kadlec, K., A.T. Feßler, T. Hauschild, and S. Schwarz. 2012. "Novel and Uncommon Antimicrobial Resistance Genes in Livestock-Associated Methicillin-Resistant Staphylococcus Aureus." Clinical Microbiology and Infection 18 (8): 745-55. doi:10.1111/j.1469-0691.2012.03842.x.

Kim, O.-S., Y.-J. Cho, K. Lee, S.-H. Yoon, M. Kim, H. Na, S.-C. Park, et al. 2012. "Introducing EzTaxon-E: A Prokaryotic 16S rRNA Gene Sequence Database with Phylotypes That Represent Uncultured Species." International Journal Of Systematic And Evolutionary Microbiology 62 (Pt 3): 716-21. doi:10.1099/ijs.0. 038075-0.

Klindworth, Anna, Elmar Pruesse, Timmy Schweer, Jörg Peplies, Christian Quast, Matthias Horn, and Frank Oliver Glöckner. 2013. "Evaluation of General 16S Ribosomal RNA Gene PCR Primers for Classical and next-Generation SequencingBased Diversity Studies.” Nucleic Acids Research 41 (1): e1-e1. doi:10.1093/ nar/gks808.

Loy, A., F. Maixner, M. Wagner, and M. Horn. 2007. "probeBase--an Online Resource for rRNA-Targeted Oligonucleotide Probes: New Features 2007." Nucleic Acids Research 35 (Database): D800-804. doi:10.1093/nar/gk1856.

Messina, Julia A., Joshua T. Thaden, Batu K. Sharma-Kuinkel, and Vance G. Fowler. 2016. "Impact of Bacterial and Human Genetic Variation on Staphylococcus Aureus Infections.” Edited by Virginia L. Miller. PLOS Pathogens 12 (1): e1005330. doi:10.1371/journal.ppat.1005330.

Muyzer G, A, Teske C.O, Wirsen, Jannasch H.W 1995. "Phylogenetic relationships of Thiomicrospira species and their identification in deep-sea hydrothermal vent samples by denaturing gradient gel electrophoresis of 16S rDNA fragments." Arch. Microbiol. 164, 165-172 (1995).

Namvar, Amirmorteza Ebrahimzadeh, Sara Bastarahang, Niloufar Abbasi, Ghazaleh Sheikhi Ghehi, Sara Farhadbakhtiarian, Parastoo Arezi, Mahsa Hosseini, Sholeh Zaeemi Baravati, Zahra Jokar, and Sara Ganji Chermahin. 2014. "Clinical Characteristics of Staphylococcus Epidermidis: A Systematic Review." GMS Hygiene \& Infection Control 9 (3).

Nyasulu, Peter, John Chipolombwe, Estée Török, and Nontombi Mbelle. 2016. "Methicillin-Resistant Staphylococcus Aureus Multiple Sites Surveillance: A Systemic Review of the Literature." Infection and Drug Resistance, February, 35. doi:10.2147/IDR.S95372.

Oliveira, Fernando, and Nuno Cerca. 2013. "Antibiotic Resistance and Biofilm Formation Ability among Coagulase-Negative Staphylococci in Healthy Individuals from Portugal." J Antibiot 66 (12): 739-41.

Petti, C. A., K. E. Simmon, J. M. Miro, B. Hoen, F. Marco, V. H. Chu, E. Athan, et al. 2008. "Genotypic Diversity of Coagulase-Negative Staphylococci Causing Endocarditis: A Global Perspective." Journal of Clinical Microbiology 46 (5): 178084. doi:10.1128/JCM.02405-07. 
Podkowik, M., J.Y. Park, K.S. Seo, J. Bystron, and J. Bania. 2013. "Enterotoxigenic Potential of Coagulase-Negative Staphylococci." International Journal of Food Microbiology 163 (1): 34-40. doi:10.1016/j.ijfoodmicro.2013.02.005.

Ruimy, Raymond, Cécile Angebault, Félix Djossou, Claire Dupont, Loïc Epelboin, Sophie Jarraud, Laurence Armand Lefevre, et al. 2010. "Are Host Genetics the Predominant Determinant of Persistent Nasal Staphylococcus Aureus Carriage in Humans?” The Journal of Infectious Diseases 202 (6): 924-34. doi:10.1086/655901.

Shore, Anna C., and David C. Coleman. 2013. "Staphylococcal Cassette Chromosome Mec: Recent Advances and New Insights." International Journal of Medical Microbiology 303 (6-7): 350-59. doi:10.1016/j.ijmm.2013.02.002.

Szymanska, Grazyna, Magdalena Szemraj, and Eligia M. Szewczyk. 2011. "SpeciesSpecific Sensitivity of Coagulase-Negative Staphylococci to Single Antibiotics and Their Combinations." Polish Journal of Microbiology 60 (2): 155-61.

Takahashi, Tatsufumi, Itona Satoh, and Naoya Kikuchi. 1999. "Phylogenetic Relationships of 38 Taxa of the Genus Staphylococcus Based on 16s rRNA Gene Sequence Analysis." International Journal of Systematic and Evolutionary Microbiology 49 (2): 725-28.

WiderströM, Micael, Johan WiströM, Elin Ek, HeLÉn Edebro, and Tor Monsen. 2011. "Near Absence of Methicillin-Resistance and Pronounced Genetic Diversity among Staphylococcus Epidermidis Isolated from Healthy Persons in Northern Sweden: Diversity Of Community S. epidermidis." APMIS 119 (8): 505-12. doi:10.1111/j.1600-0463.2011.02757.x.

Witte, W., Cuny, C., Klare, I., Nübel, U., Strommenger, B., \& Werner, G. (n.d.) 2008. Emergence and spread of antibiotic-resistant Gram-positive bacterial pathogens, 298(5), 365-377. http://doi.org/10.1016/j.ijmm.2007.10.005. 